\title{
On the Spatial Velocity Electrocardiogram
}

\section{Clinical Application}

Toyomi Sano, M.D., Fumio Suzuki, M.D., Tadayuki Hiroki, M.D., and Tohru SAWANobori, M.D.

\section{SUMmary}

Employing the apparatus which we made of analog computers, spatial velocity electrocardiograms were taken from 321 cases composed of 54 normal cases, 71 cases of left ventricular hypertrophy, 12 cases of right ventricular hypertrophy, 14 cases of complete left bundle branch block, 30 cases of complete right bundle branch block, 10 cases of incomplete right bundle branch block, 41 cases of myocardial infarction and 89 cases of ventricular premature beats. The normal sVECG was composed of 8 chief deflections: $\pi_{1}$ and $\pi_{2}$ as the counterpart of the $\mathrm{P}$ wave of the electrocardiogram, $\xi, \rho_{1}, \rho_{2}$ and $\sigma$ as that of the QRS, and $\tau_{1}$ and $\tau_{2}$ as that of the T wave. Normally $\rho_{2}$ and $\tau_{2}$ were larger than $\rho_{1}$ and $\tau_{1}$, respectively. In ventricular hypertrophy $\xi_{\rho \sigma}$ tended to show more splitting and $\tau$ to be flatter or the ratio $\tau_{1} / \tau_{2}$ was smaller than normal. In left bundle branch block splitting of $\xi \rho \sigma$ was most marked and in right bundle branch block $\sigma$ was wide, frequently making a separate $\sigma^{\prime}$. In myocardial infarction $\xi$ was wide occasionally, and $\sigma$ was usually wide. $\rho_{1}$ was often larger than $\rho_{2}$ in anterior myocardial infarction and was smaller than $\rho_{2}$ in posterior myocardial infarction. $\tau$ was variously abnormal in most cases. These and other findings were found useful for diagnosis and the sVECG was found to be especially useful when combined with the vectorcardiogram by effectively supplementing its weak points.

\section{Additional Indexing Words :}

Analog computer Vectorcardiograms by Frank's lead system Left ventricular hypertrophy Right ventricular hypertrophy Left bundle branch block Right bundle branch block Myocardial infarction Ventricular premature beats First derivative electrocardiogram

$7 \mathrm{HE}$ spatial velocity electrocardiogram (sVECG) expresses the spatial velocity of inscription of vector loops of the vectorcardiogram. It is obtained from vectorcardiograms or electrocardiograms by computation of the following equation:

$$
\mathrm{sV}=\sqrt{\left(\frac{d x}{d t}\right)^{2}+\left(\frac{d y}{d t}\right)^{2}+\left(\frac{d z}{d t}\right)^{2}}
$$

where $x, y, z$ are components in 3 orthogonal axes of the vectorcardiogram.

From the Institute for Cardiovascular Diseases, Tokyo Medical and Dental University, Tokyo. Received for publication September 23, 1967. 
In our previous report ${ }^{11}$ an apparatus made of analog computers was described with automatically computes this equation and records the curve expressing the result. Employing this apparatus, a number of sVECG were recorded. In this report their features of normal and abnormal entities are described.

\section{Methods and Materials}

Vectorcardiograms were obtained by Frank's lead system ${ }^{2)}$ from 54 cases of normal subjects, 71 cases of left ventricular hypertrophy, 12 cases of right ventricular hypertrophy, 14 cases of complete left bundle branch block, 30 cases of complete right bundle branch block, 10 cases of incomplete right bundle branch block, 41 cases of myocardial infarction and 89 cases of ventricular premature beats. The component corrected electrocardiograms were led to the spatial velocity electrocardiograph either directly or after recording by the analog tape recorder. Spatial velocity electrocardiograms were thus obtained from 321 cases in total. They were recorded by a heat stylus direct writing electrocardiograph at a speed of $50 \mathrm{~mm}$. $/ \mathrm{sec}$. Usually 2 kinds of sVECG were taken in each case, one for analysis of the QRS complexes, employing the R-C combination with time constant of $2 \mathrm{msec}$. of the differentiator of the apparatus, and another for analysis of the other slow components such as $P$. and $\mathrm{T}$ waves, employing that with the time constant of $15 \mathrm{msec}$.

\section{RESULTS}

1. Normal The normal sVEGG is usually composed of 8 main deflections. These deflections are provisionally called $\xi, \rho_{1}, \rho_{2}$ and $\sigma$ corresponding to the QRS complexes of the ordinary electrocardiogram, $\tau_{1}$ and $\tau_{2}$ corresponding to the $\mathrm{T}$ wave, and $\pi_{1}$ and $\pi_{2}$ corresponding to the $\mathrm{P}$ wave (Fig. 1 ). Among 54 normal records $\rho_{2}$ was larger than $\rho_{1}$ in 46 cases $(85.2 \%)$ and was equal to $\rho_{1}$ in 6 cases $(11.1 \%$ ) (Table I). $\sigma$ was larger than $\xi$ in 49 cases $(90.7 \%) . \tau_{2}$ was larger than $\tau_{1}$ in all 54 cases, and the ratio $\tau_{1} / \tau_{2}$ was $0.44 \pm 0.16$ (S.D.). Therefore, normal findings of the ventricular complexes were uniform and its feature can be summarized as $\rho_{2}>\rho_{1}, \sigma>\xi$ and $\tau_{2}>\tau_{1}$ and shown as in the second row of Fig. 1. This means that in the midportion of the QRS and the $\mathrm{T}$ loop inscription is made at a low speed, each minimum producing $\rho_{2}$ and $\rho_{1}$ and $\tau_{2}$ and $\tau_{1}$ and that the velocity of the afferent limb of the QRS and T loop is normally larger than that of their efferent $\operatorname{limb}\left(\rho_{2}>\rho_{1}, \tau_{2}>\tau_{1}\right)$. At the beginning and terminal portion of the QRS loop the speed of inscription is slightly different from the midportion, and the latter is larger than the former $(\sigma>\xi)$.

2. Left ventricular hypertrophy Main features of sVECG of left ventricular hypertrophy were a prominent splitting of $\xi \rho \sigma$ and flattening of $\tau$ or a decrease of $\tau_{1} / \tau_{2}$ ratio. But depending upon the severity of left ventricular hypertrophy, a variety of findings was experienced. Two types of $\xi \rho \sigma$ were observed. 


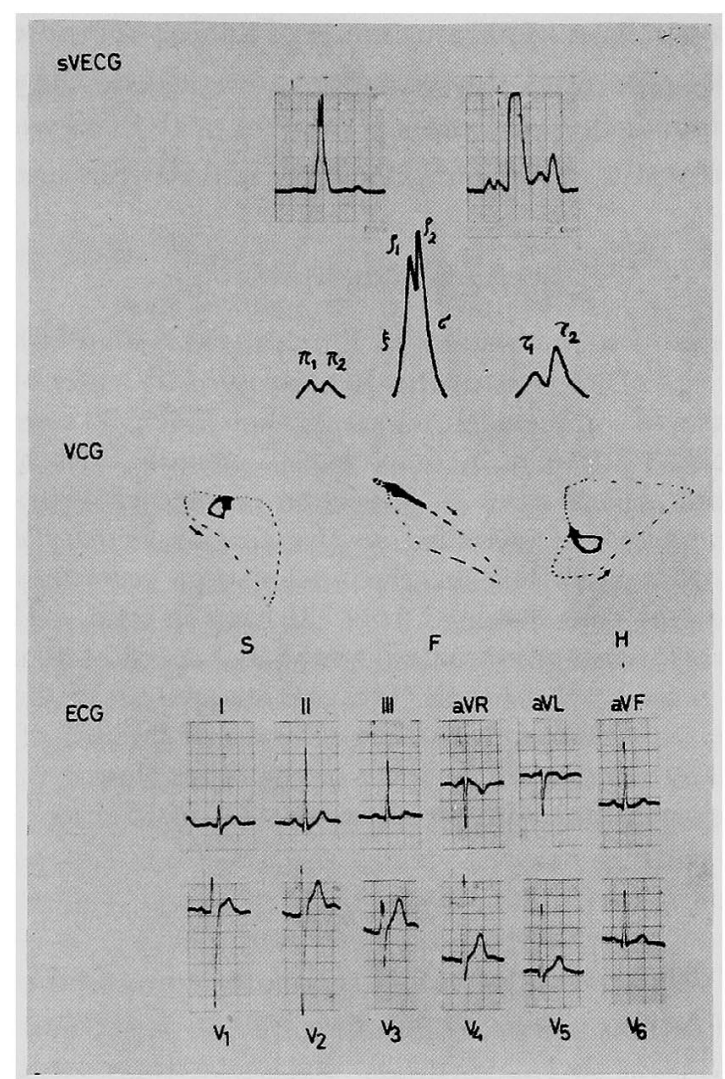

Fig. 1. A spatial velocity electrocardiogram of a normal case. In sVECG the upper left figure was taken with time constant of 2 msec. to show $\xi \rho \sigma$ and the upper right figure with time constant of $15 \mathrm{msec}$. to show $\pi$ and $\tau$. The lower figures were tracings for explanation. A vectorcardiogram and an electrocardiogram taken from the same subject are shown in the middle and lower part.

S: left sagittal plane; F : frontal plane; H: horizontal plane

Table I. sVECG Configuration of Normal Cases

\begin{tabular}{c|ccc|ccc|ccc|c}
\hline & $\rho_{1}<\rho_{2}$ & $\rho_{1} \stackrel{\rho}{=} \rho_{2}$ & $\rho_{1}>\rho_{2}$ & $\xi<\sigma$ & $\begin{array}{c}\xi \& \sigma \\
\xi=\sigma\end{array}$ & $\xi>\sigma$ & $\tau_{1}<\tau_{2}$ & $\tau_{1}=\tau_{2}$ & $\tau_{1}>\tau_{2}$ & Total \\
\hline $\begin{array}{c}\text { No. of } \\
\text { Subjects } \\
(\%)\end{array}$ & 46 & 6 & 2 & 49 & 0 & 5 & 54 & 0 & 0 & 54 \\
$(85.2)$ & $(11.1)$ & $(3.7)$ & $(90.7)$ & $(0)$ & $(9.3)$ & $(100)$ & $(0)$ & $(0)$ &
\end{tabular}

Type I $\xi \rho \sigma$ had a normal configuration and was found in 40 of 71 cases of left ventricular hypertrophy $(56.3 \%)$. Type II $\xi \rho \sigma$ showed a more or less prominent split and was found in 31 cases $(43.7 \%$ ) (Table IIA). As for $\tau, 3$ types were observed. Type I $\tau$ had a normal configuration and was found in 34 cases $(47.8 \%)$. Type II $\tau$ was flat and was found in 13 cases $(18.4 \%)$. 


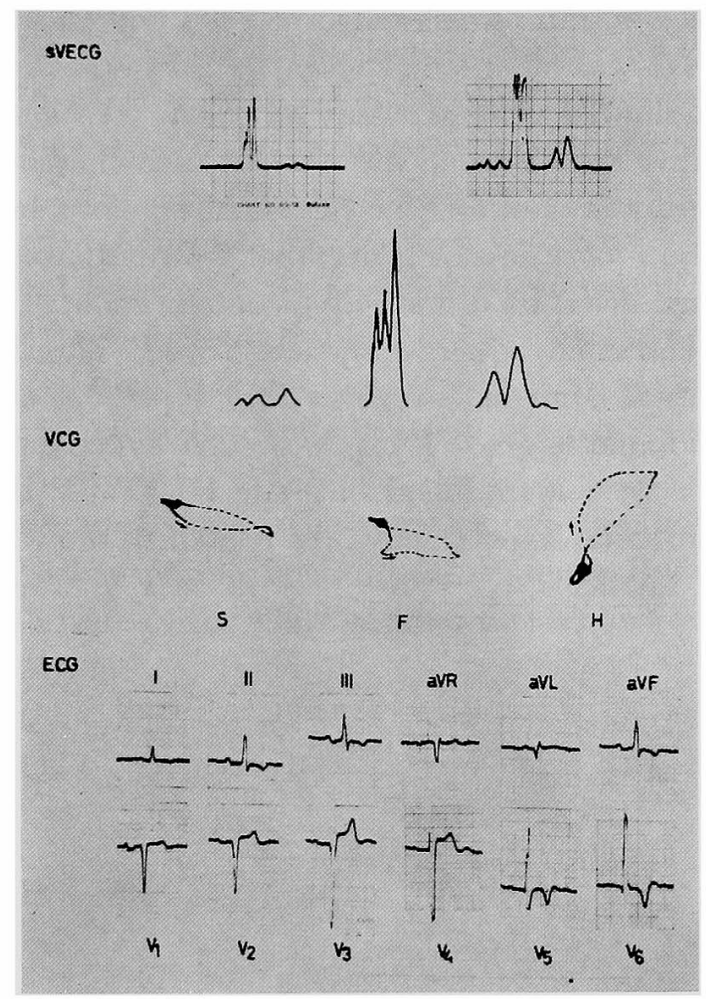

Fig. 2. A spatial velocity electrocardiogram of a case of left ventricular hypertrophy.

Figure arrangement is the same with Fig. 1.

Table II. sVEGG Configuration of Ventricular Hypertrophy

A

\begin{tabular}{|c|c|c|c|c|c|c|}
\hline & I & II & I & $\begin{array}{c}\tau \\
\text { II }\end{array}$ & III & Total \\
\hline LVH & $\begin{array}{c}40 \\
(56.3)\end{array}$ & $\begin{array}{c}31 \\
(43.7)\end{array}$ & $\begin{array}{c}34 \\
(47.8)\end{array}$ & $\begin{array}{c}13 \\
(18.4)\end{array}$ & $\begin{array}{c}24 \\
(33.8)\end{array}$ & 71 \\
\hline $\mathrm{RVH}$ & $\begin{array}{c}3 \\
(25)\end{array}$ & $\begin{array}{c}9 \\
(75)\end{array}$ & $\begin{array}{c}3 \\
(25)\end{array}$ & $\begin{array}{c}6 \\
(50)\end{array}$ & $\begin{array}{c}3 \\
(25)\end{array}$ & 12 \\
\hline
\end{tabular}

B

\begin{tabular}{|c|c|c|c|c|c|c|}
\hline $\begin{array}{l}\text { LVH } \\
\text { Grade }\end{array}$ & I & II & I & $\tau$ & III & Total \\
\hline I & 19 & 7 & 14 & 10 & 2 & 26 \\
\hline II & 13 & 9 & 14 & 3 & 5 & 22 \\
\hline III & 8 & 15 & 6 & 0 & 17 & 23 \\
\hline
\end{tabular}

Figures show number of cases, per cent incidence of which is shown in parenthesis. LVH : left ventricular hypertrophy;

$\mathrm{RVH}$ : right ventricular hypertrophy. 
Type III $\tau$ had a $\tau_{1} / \tau_{2}$ ratio smaller than normal and was found in 24 cases $(33.8 \%)$. Type III was often found when the corresponding $\mathrm{T}$ wave of the electrocardiogram showed a characteristic inversion, as shown in Fig. 2. Since there are no negative deflections in the sVECG, such characteristic features of the electrocardiogram are lost to some extent. Therefore, not only a normallike $\mathrm{T}$ wave but also a more advanced negative $\mathrm{T}$ wave showed type $\mathrm{I} \tau$, when it had a mirror image inversion of normal $\mathrm{T}$ configuration.

When left ventricular hypertrophy was divided into 3 grades depending upon its severity as Sano and associates did, ${ }^{3)}$ type I $\xi \rho \sigma$ and types I and II $\tau$ were more often found in grade I left ventricular hypertrophy, while type II $\xi \rho \sigma$ and type III $\tau$ were more found in grade III (Table IIB). Namely, in advanced left ventricular hypertrophy, $\xi \rho \sigma$ tended to show a prominent split and $\tau$ to show a smaller $\tau_{1} / \tau_{2}$.

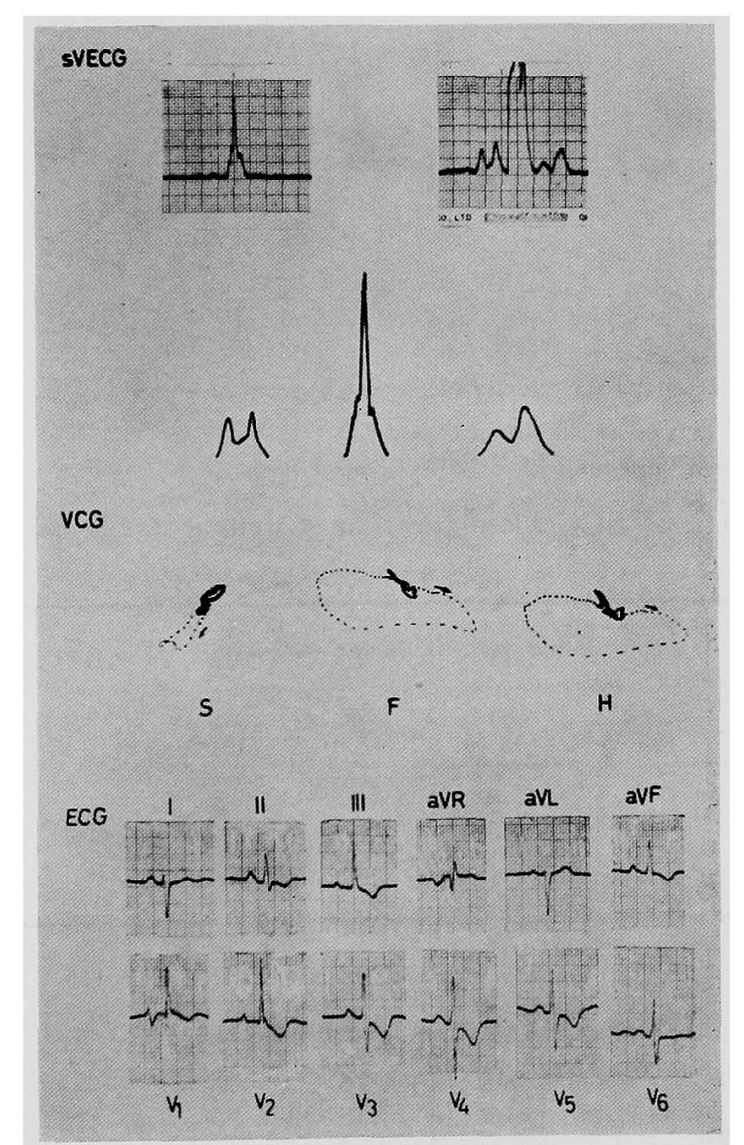

Fig. 3. A spatial velocity electrocardiogram of a case of right ventricular hypertrophy,

Figure arrangement is the same with Fig. 1. 
3. Right ventricular hypertrophy Twelve cases of right ventricular hypertrophy were classified in a way similar to left ventricular hypertrophy and are shown in Table IIA. Splitting of $\xi \rho \sigma$ was observed in more cases with right ventricular hypertrophy than with left ventricular hypertrophy and 9 cases belonged to type II $\xi \rho \sigma(75 \%)$. In contrast to left ventricular hypertrophy, $\rho_{1}$ was frequently larger than $\rho_{2}$ in right ventricular hypertrophy. $\tau$ was more or less similar to that of left ventricular hypertrophy except that $\tau_{2}$ did not become so large (Fig. 3), but type distribution in right ventricular hypertrophy was quite different from that in left ventricular hypertrophy in $\tau$ types as well as $\xi \rho \sigma$ types. The reason might be due to the limited number of cases of right ventricular hypertrophy in this study.

4. Left bundle branch block In complete left bundle branch block $\xi \rho \sigma$ showed most prominent splitting in addition to its longer duration than $0.12 \mathrm{sec}$. Mostly there was a wide nadir, which separated two chief deflections of $\rho_{1}$ and

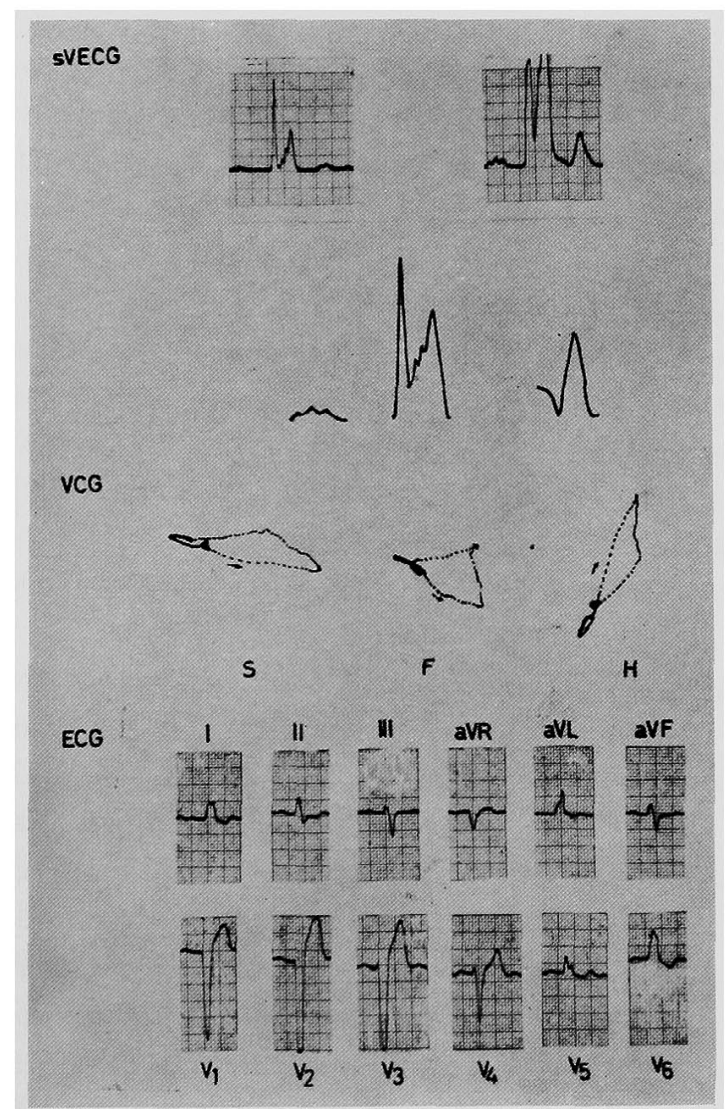

Fig. 4. A spatial velocity electrocardiogram of a case of left bundle branch block.

Figure arrangement is the same with Fig. 1. 
$\rho_{2}$ (Fig. 4). $\quad \rho_{1}$ was larger than $\rho_{2}$ in 13 of 14 cases of left bundle branch block $(92.9 \%)$ except for one case, where $\rho_{1}$ was smaller than $\rho_{2}$. Neither $\xi$ nor $\sigma$ was often differentiated. Mostly $\tau_{1}$ fused with the preceding $\sigma$. The ratio of $\tau_{1} / \tau_{2}$ was usually smaller than that of normal cases as in cases of marked left ventricular hypertrophy.

5. Right bundle branch block

(a) Complete right bundle branch block. The prominent sVECG features of right bundle branch block lie in a terminal deflection of $\xi \rho \sigma$, which we designated as $\sigma^{\prime}$ (Fig. 5). It was more often a more or less separate, distinct deflection after the usual $\xi \rho \sigma$ and was called provisionally $\sigma^{\prime}$. In type I another $\sigma$ was found in addition to $\sigma^{\prime}$. This type I was found in 21 of 30 cases of right bundle branch block $(70.0 \%)$. In other cases there was only $\sigma^{\prime}$ and no other $\sigma$ could be found. This type II was observed in 9 cases $(30.0 \%)$. The duration of $\sigma$ or $\sigma^{\prime}$ of complete right bundle branch block was longer than 45

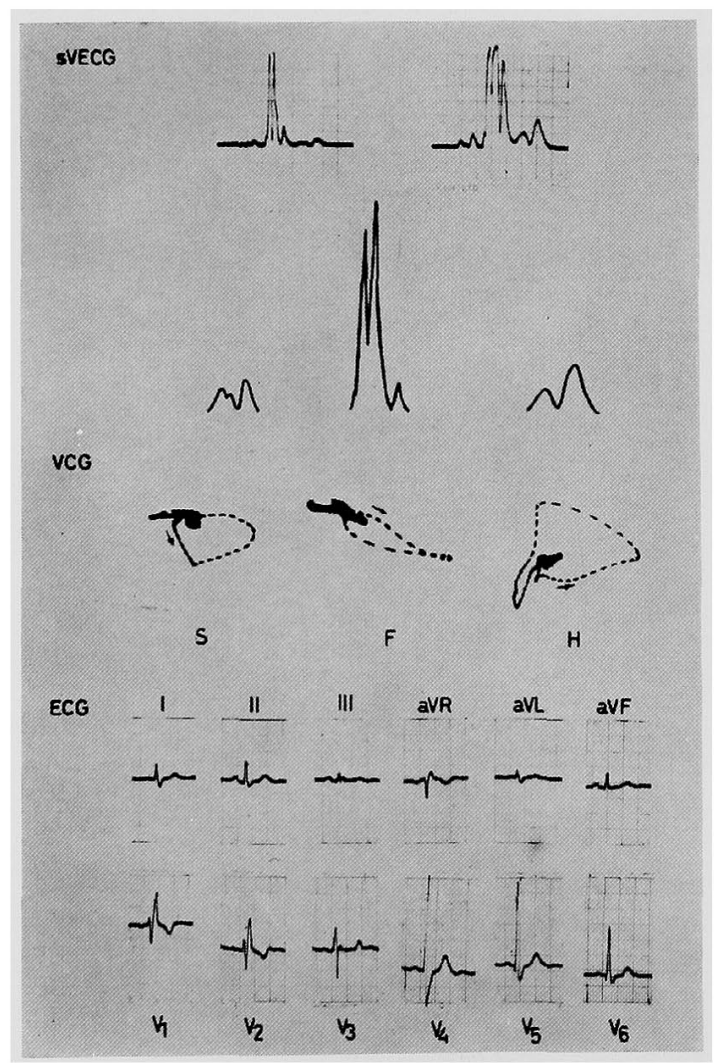

Fig. 5. A spatial velocity electrocardiogram of a case of right bundle branch block.

Figure arrangement is the same with Fig. 1. 
Table III. sVECG Configuration of Right Bundle Branch Block

A

\begin{tabular}{ccc|cc|c}
\hline I & II & III & I & II & Total \\
\hline 22 & 7 & 1 & 21 & 9 & 30 \\
$(73.4)$ & $(23,3)$ & $(3.3)$ & $(70.0)$ & $(30,0)$ &
\end{tabular}

B

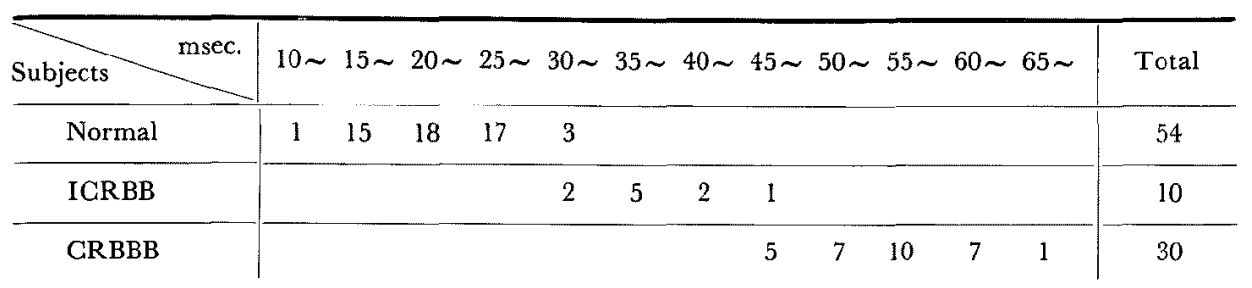

A : Features of complete right bundle branch block

B : Duration of $\sigma$ or $\sigma^{\prime}$ in right bundle branch block

ICRBBB : incomplete right bundle branch block

CRBBB : complete right bundle branch block.

msec. in contrast to the normal range of $15 \mathrm{msec}$. to $30 \mathrm{msec}$. (Table IIIB). Such terminal deflection corresponded to the terminal appendage of the QRS loop.

The rest of $\xi \rho \sigma$, namely $\xi \rho$ can be divided into 3 types. In type $I$ this resembled the normal $\xi \rho$. This type was found in 22 of 30 cases $(73.4 \%)$. In type II $\rho_{1}$ was larger than $\rho_{2}$. This type was found in 7 cases $(23.3 \%)$. In type III $\xi_{\rho}$ had a marked splitting. This type was exceptional, being found only in one case $(3.3 \%)$. Since this case was combined with myocardial infarction, this finding may not have been due to right bundle branch block.

The ratio of $\tau_{1} / \tau_{2}$ was smaller than normal as in left ventricular hypertrophy.

(b) Incomplete right bundle branch block. The $\xi \rho \sigma$ of incomplete right bundle branch block differed from normal only in its wider $\sigma$. As is shown in Table IIIB, the duration of $\sigma$ of 10 cases of incomplete right bundle branch block was from $30 \mathrm{msec}$. to 45 . msec.

No difference could be found in $\tau$ of incomplete right bundle branch block from normal, as far as this series was concerned.

6. Myocardial infarction Since the presence of abnormal $Q$ waves is pathognomonic for myocardial infarction, we expected to find an abnormal $\xi$ in this state. Actually we found an abnoramally large and wide $\xi$ as a prominent feature (Fig. 6), but not in all cases of myocardial infarction. Abnormal $\xi$ was found in 7 of 22 cases of anterior infarction $(31.8 \%)$ and in 5 of 19 cases of posterior infarction (26.3\%), as is shown in Table IV. 


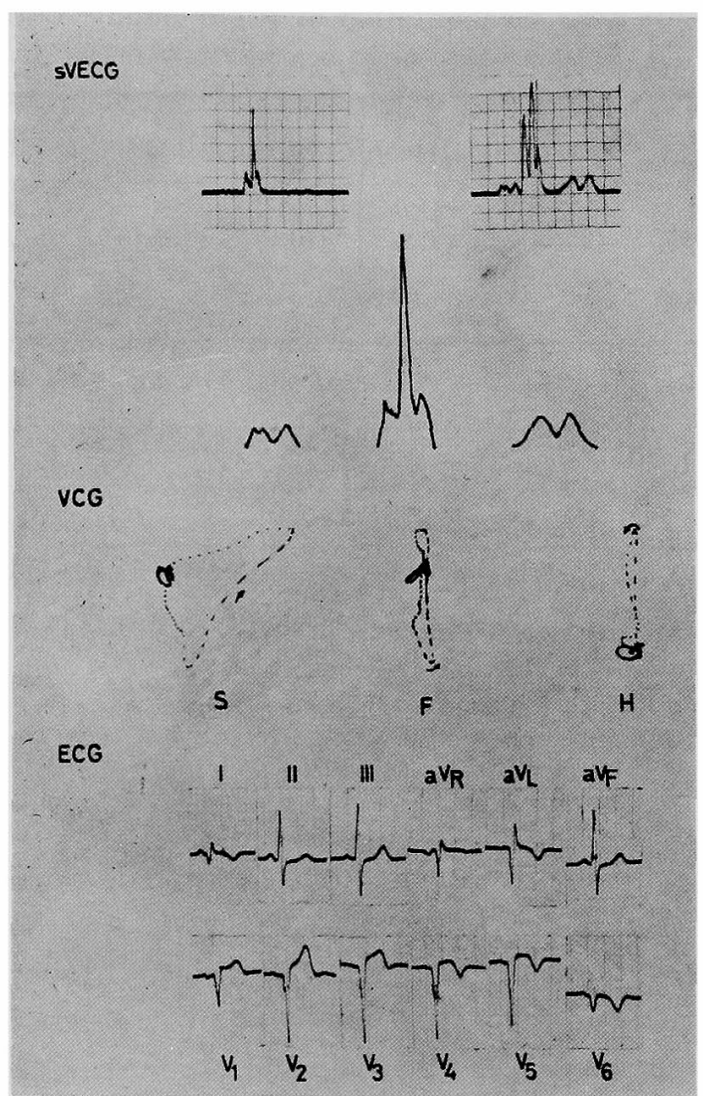

Fig. 6. A spatial velocity electrocardiogram of a case of myocardial infarction.

Figure arrangement is the same with Fig. 1.

Table IV. sVECG Configuration of Myocardial Infarction

\begin{tabular}{|c|c|c|c|c|c|c|c|c|c|}
\hline & $\xi$ & & $\rho$ & & $\sigma$ & & $\tau$ & & \multirow{2}{*}{ Total } \\
\hline & $\begin{array}{c}\text { Abnormal } \xi \\
+\quad-\end{array}$ & \multicolumn{3}{|c|}{$\rho_{1}<\rho_{2} \quad \rho_{1}=\rho_{2} \quad \rho_{1}>\rho_{2}$} & $\begin{array}{c}\text { Abnormal } \sigma \\
+\quad-\end{array}$ & $\tau_{1}<\tau_{2}$ & $\tau_{1}=\tau_{2}$ & $\tau_{1}>\sigma_{2}$ & \\
\hline $\begin{array}{l}\text { Anterior } \\
\text { Infarction }\end{array}$ & $\begin{array}{cc}7 & 15 \\
(31.8) & (68.2)\end{array}$ & $\begin{array}{c}3 \\
(13.6)\end{array}$ & $\stackrel{1}{(4.6)}$ & $\begin{array}{c}18 \\
(81.8)\end{array}$ & $\begin{array}{cc}10 & 12 \\
(45.5) & (54.5)\end{array}$ & $\begin{array}{c}1 \\
(4.6)\end{array}$ & $\begin{array}{c}3 \\
(13.6)\end{array}$ & $\begin{array}{c}18 \\
(81.8)\end{array}$ & 22 \\
\hline $\begin{array}{l}\text { Posterior } \\
\text { Infarction }\end{array}$ & $\left(\begin{array}{cc}5 & 14 \\
(26.3) & (73.7)\end{array}\right.$ & $\begin{array}{c}15 \\
(78.9)\end{array}$ & $\begin{array}{c}0 \\
(0)\end{array}$ & $\begin{array}{c}4 \\
(21.1)\end{array}$ & $\left(\begin{array}{cc}12 & 7 \\
(63.5) & (36.5)\end{array}\right.$ & $\begin{array}{c}1 \\
(5.3)\end{array}$ & $\begin{array}{c}3 \\
(15.8)\end{array}$ & $\begin{array}{c}15 \\
(78.9)\end{array}$ & 19 \\
\hline
\end{tabular}

However, it was noteworthy that an abnormal $\rho$ relation was found: namely, in 18 cases of anterior infarction $\rho_{1}$ was larger than $\rho_{2}(81.8 \%)$. In contrast in 15 cases of posterior infarction $\rho_{1}$ was smaller than $\rho_{2}(78.9 \%)$. Furthermore, $\sigma$ was frequently wider, and of more than $30 \mathrm{msec}$. duration in contrast to the normal value less than $30 \mathrm{msec}$. This wide $\sigma$ was found in 10 cases 
of anterior infarction (45.5\%) and 12 cases of posterior infarction $(63.5 \%)$. This phenomenon was observed even when the criteria of Grant ${ }^{4)}$ on periinfarction block were not fulfilled. In this report only cases of old infarction were analysed. But $\tau$ showed already abnormal relations. When the electrocardiogram showed a typical coronary $\mathrm{T}$ wave, $\tau_{1}$ was mostly equal to $\tau_{2}$ (Fig. 6). This relation was found in 3 cases of anterior infarction (13.6\%) and also in 3 cases of posterior infarction $(15.8 \%$ ). In most of other cases an abnormal $\tau$ relation, where $\tau_{1}$ was larger than $\tau_{2}$, was found in 18 cases of anterior infarction $(81.8 \%)$ and in 15 cases of posterior infarction $(78.9 \%)$. Occasionally 3 deflections, $\tau_{1}, \tau_{2}$ and $\tau_{3}$, were observed.

Therefore, although $\xi$ finding was not as pathognomonic as was expected from the eletrocardiogram, many other sVEGG findings, which usually are unnoticed in the ordinary electrocardiogram, were found to be diagnostically useful.

7. Ventricular premature beats In the electrocardiogram QRS complexes of ventricular premature beats resemble either those of left bundle branch block or of right bundle branch block. But in the sVEGG $\xi \rho \sigma$ of ventricular premature beats did not always resemble that of bundle branch block. Usually $\xi \rho \sigma$ had a more or less marked splitting, and each $\xi, \rho$ and $\sigma$ could not be easily identified. The $\xi \rho \sigma$ of ventricular premature beats can be divided into 5 types. In type $I$ the initial half of $\xi \rho \sigma$ was smaller than its terminal half. This type was found in 27 of 89 cases of ventricular premature beats $(30 \%)$. In type II the initial half of $\xi \rho \sigma$ was larger than its terminal half. This type resembled $\xi \rho \sigma$ of left bundle branch block and was found in 15 cases (17\%). Type III $\xi \rho \sigma$ was small in all deflections and was found in 11 cases $(12 \%)$. Type IV $\xi \rho \sigma$ had a larger midportion and was found in 10 cases $(11 \%)$. Type $\mathrm{V} \xi \rho \sigma$ included all other forms of $\xi \rho \sigma$ which could not be classified into any of the above-mentioned 4 types and was found in 26 cases (29\%).

The $\tau$ resembled mostly that of left bundle branch block in all types and the $\tau_{1} / \tau_{2}$ ratio was much smaller than normal.

Clinical evaluation of these types will be reported elsewhere. ${ }^{5)}$

8. $\sigma-\tau$ segment One of the weak points of the vectorcardiogram is inadequate information on the ST-T contour of the electrocardiogram. The sVECG is expected to give more detailed and more effective information than the ordinary electrocardiogram because of its first derivative components. As a matter of fact, its $\sigma-\tau$ segment was found to show many varieties with corresponding clinical significance. Not only the ratio of $\tau_{1} / \tau_{2}$, but also the configuration and time position of peaks of $\tau_{1}$ and $\tau_{2}$ and of the nadir between them seemed to be important. Unfortunately our present method of heat stylus recording has the disadvantage of inertia. When there was a large stylus move- 
ment in writing $\xi \rho \sigma$, the early $\sigma-\tau$ portion might not be inscribed faithfully, even though a low time constant was adopted (Fig. 4). The $\sigma \tau-\tau$ problem is now being restudied using a cathode-ray-oscilloscope.

\section{Discussion}

Several years ago Sayers et al.6) and Abildskov et al. ${ }^{71}$ proposed transformation of EGG data into curves of spatial magnitude and orientation. Hellerstein and Hamlin ${ }^{81}$ and Yano and Pipberger ${ }^{9 /}$ proposed similar transformation into spatial velocity in addition. Since they were interested in so many factors, no inclusive and intensive study, particularly on sVECG, seems to have appeared as yet. Informations on spatial magnitude and orientation were included in ordinary VGG, and were known to some extent. It was sVECG among them that was mostly unknown and needed a further evaluation.

Simonson and his associates ${ }^{15)}$ reported that the angular speed in the central segment of the spatial QRS loop was smaller in 26 patients with ventricular hypertrophy, myocardial infarction and bundle branch block than in 103 normal men. In contrast, Yano and Pepberger") made an analysis of sVECG, employing a digital computer, and concluded that they could not find any special information useful for differentiation of each pathological entity. However, since they employed a digital computer, noncontinuous sampling of ECG was inevitable. Actually they sampled at 10 equal intervals during the QRS complex. This sampling and their smoothing by filtering procedures of the moving average method may have lost much information. In order to avoid this defect, we made an analog instrument, and, by employing this, we were able to obtain the above-mentioned results.

It was found that even with the sVECG only the diagnosis of each pathological entity could be made in many cases. However, the most useful employment of this method is in combination with the vectorcardiogram. The sVECG supplements the defects of the vectorcardiogram more effectively than the ordinary electrocardiogram does. In the vectorcardiogram usually density of time markers supplies the information of inscription speed of the loop. Evidently this is very defective, and the sVEGG supplies this information quantitatively. The ST-T configuration of the ordinary electrocardiogram is most defective in vectorcardiographic presentation, but is expressed effectively in the sVECG, which will be reported elsewhere. The analysis of arrhythmia as well as duration of each part including the counterparts of the PQ interval and QT interval can, of course, be made with the sVECG with ease. It is possible, therefore, that combining the use of the sVECG and the vectorcardiogram surpasses widely the ordinary electorcardiogram in diagnostic usefulness. 
In this study we used a direct wirting heat stylus recorder. Langner and associates ${ }^{11}$ reported the diagnostic usefulness of the first derivative electrocardiogram for myocardial infarction by recording high frequency components up to $600 \mathrm{c} / \mathrm{s}$. If this is true, since the sVECG includes differential components, it may provide such information by employing a suitable recorder.

\section{REFERENCES}

1. Sano, T., Suzuki, F., Takahashi, T., Fukamachi, M., and Furukawa, T.: Jap. Heart J. 8: 301, 1967.

2. Frank, E.: Circulation 13: 737, 1956.

3. Sano, T., Tsuchihashi, H., Takigawa, S., and Shimamoto, T.: Am. Heart J. 61: 802, 1961.

4. Grant, R. P.: Prog. Cardiovas. Dis. 2: 237, 1959-1960.

5. Sano, T., Suzuki, F., Hiroki, T., and Sato, S.: Proc. Japan Acad. 43: 812, 1967

6. Sayers, B. McA., Silberberg, F. G., and Durie, D. F.: Am. Heart J. 49: 323, 1955.

7. Abildskov, J. A., Ingerson, W. E., and Hisey, B. L.: Circulation 14: 556, 1956.

8. Hellerstein, H. K. and Hamlin, R.: Am. J. Cardiol. 6: 1049, 1960.

9. Yano, K. and Pipberger, H. V.: Circulation 29: 107, 1964.

10. Simonson, E., Schmitt, O. H., Blackburn, H. W., and Levine, R. B.: Circulat. Res. 3: 409, 1955.

11. Langner, P. H., Geselowitz, D. B., and Mansure, F. T.: Am. Heart J. 62: 746, 1961. 